\title{
The effect of irrelevant information in a multidimensional oddity task'
}

DANIEL S. LORDAHL

UNIVERSITY OF MIAMI

Two studies were conducted using oddity problems with geometric designs as stimuli. The number of bi-leveled dimensions in the stimuli was either 3, 5, or 7, with one dimension relevant, for the major variable in each study. In the first study, the relevant dimension was varied, while in the other study only one relevant dimension was employed. Trials to criterion were affected by the relevant dimension variable in the first case and by the number of irrelevant dimensions in the second.

The intention of the line of research illustrated in the present studies is to measure hypothesis behavior in humans and find variables which affect the rate and kind of such behavior. In an earlier study (Lordahl, 1965), the present method of limited knowledge of results was shown to permit the assessment of the degree to which the specific stimulus dimensions are tested with the oddity principle by Ss. Specifically, the present studies were conducted to allow the rate and kind of oddity hypotheses to be measured as a function of the number of stimulus dimensions varied in the problems.

\section{Method}

Stimuli and task. On any given trial, $\mathrm{S}$ viewed three sets of geometric designs (rear-projected onto a translucent screen) and was required to select one of the sets (patterns) by pulling one of three levers corresponding to the patterns below the screen. Figure 1 illustrates the maximum information load used in the stimuli (seven bi-leveled dimensions). These dimensions were: shading, number slope, location (top or bottom), dot, form, and border. As illustrated in Fig. 1, on each trial one of the three patterns was odd with respect to any one of the stimulus dimensions.

Ss were instructed that only one response was correct on a given trial and that the rule of solution was the oddity principle with respect to one of the dimensions. Trials were grouped into blocks of three. If and only if all three of the S's responses in a block were correct, a light was lighted to indicate this outcome to the $S$. If any error was made, no information was given to the $\mathrm{S}$. These feedback contingencies were explained to the Ss and the task was self-paced, with a blank frame presented following each block of three trials. Stimuli were arranged such that each dimension used produced a different sequence of responses, within any block of trials, when the oddity principle was applied.
Designs. In Experiment $1,3,5$, or 7 stimulus dimensions, in the order listed above, were combined with three different problems (relevant dimensions), shading, number, or location, to yield nine treatments. In Experiment II, the same conditions of 3, 5, or 7 stimulus dimensions were again used but the relevant dimension was held constant for all Ss (location always relevant). Ss were drawn from introductory psychology classes and each served in only one treatment and only one study. In Experiment I, five Ss were used in each of the nine treatments, while $20 \mathrm{Ss}$ were used in each of the three conditions of Experiment II. In the first experiment, a maximum of 28 trial blocks was given unless the criterion of three successive correct trial blocks was obtained. A maximum of 25 blocks was used in the second experiment, with the same criterion limitation.

Additional details of the procedure and task may be found in Lordahl, 1965.

\section{Results and Discussion}

In the first study, trial blocks to criterion (maximum of 28) averaged $7.40,1.27$, and 18.93 for the relevant dimensions of shading, number, and location, respectively $(F=16.89, \mathrm{df}=2 / 36, \mathrm{p}<.001)$. However, the means for number of dimensions, $7.40,9.13$, and 11.07 for 3,5 , and 7 dimensions, gave an $F$ of less than one, as did the interaction effects. The large between-Ss variability required the increased sample size found in Experiment II in order to demonstrate significant effects of irrelevant information on mean trial blocks. The differential difficulty of the problems in the first study suggested that the location dimension might be used to produce a more sensitive test of irrelevant information effects.

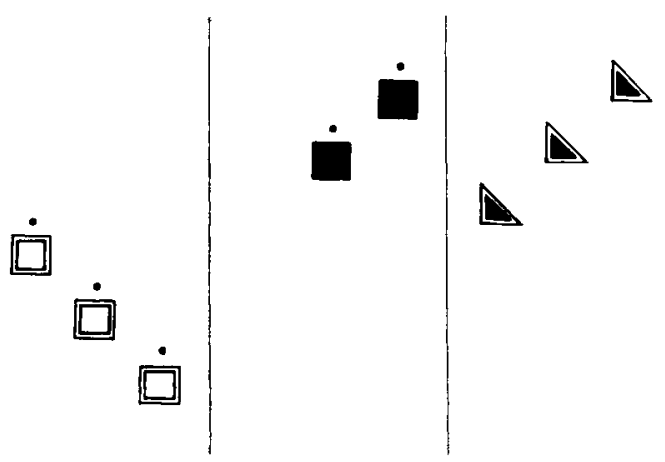

Fig. 1. An example of the 3 pattems from a 7 dimension problem. 
Table 1. Proportional Distribution of Oddity Response Sequences

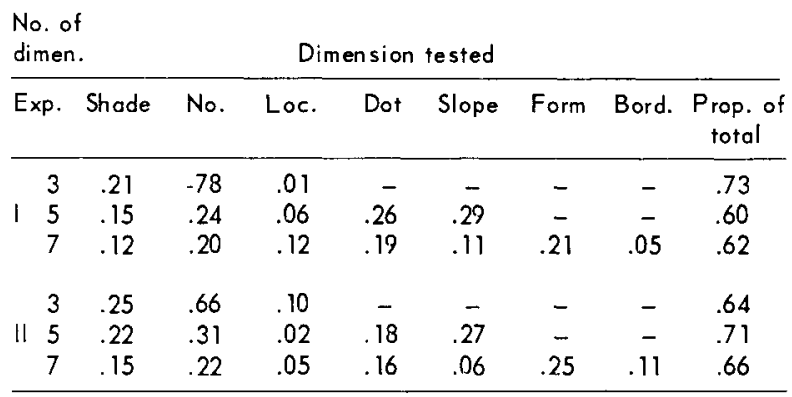

The trial block means for 3,5, and 7 dimensions in Experiment II were 18.55, 23.85, and 22.80, respectively $(F=4.03, d f=2 / 57, p<.05)$. The significance of the number of dimensions in the task is supported by the number of solutions for each group of 20 Ss: 10,2 , and 3 , respectively, for 3,5 , and 7 dimension problems. These totals and the totals for non-solvers yielded a contingency chi-sqaure ( $d f=2)$ of 11.40, which is significant beyond the .01 level. For each $\mathrm{S}$ in each study, the response sequence for each block of pre-solution trials was used to determine which dimension (if any) had been tested using the oddity principle. Since each dimension would have produced a different response sequence than the others in any trial block when so tested, the totals for each dimension tested may be used to infer the relative proportions of testing these dimensions. In Table 1, the group totals for each level of irrelevant information have been converted to proportions coinciding with the oddity response sequence for each stimulus dimension. Both studies are included in Table 1 and the proportion of oddity response sequences to total trial blocks is given for each condition in the last column of the table.

Note in Table 1 that location was a low dominance dimension. The difficulty of problem solution when location was relevant may be explained on this basis. The proportion of oddity response sequences for each S was also used to infer "random" responding. All non-oddity response sequences were defined as "random," and a correction for guessing was applied to equalize the different number of dimensions in the various conditions. When the first criterion block is included, these computations permit a "random" proportion for each $S$ to be used. The analysis of variance of these scores showed only one significant component. In the first study, the mean proportions of "random" responding for the three problems were $.135, .092$, and .316 , for shading, number, and location, respectively. The corresponding $F$ was 5.73, $p<.05$, with $\mathrm{df}=2 / 36$. Although the mean random responding increased in each experiment with increased irrelevant information, the trends were not significant.

It might be argued that the increase in number of stimulus dimensions increases difficulty of the problem simply by increasing the number of dimensions which must be tested by the Ss. However, it should also be noted that when the relevant dimension is not discovered reasonably early by the $\mathrm{Ss}$, the data show a tendency for increased "random" responding. This increase may be due to a tendency to test complex hypotheses, such as those involving combinations of dimensions, under these conditions.

The development of a set of stimuli with scaled degrees of differences between the levels of the dimensions is in progress. The ease of problem solution should be related to such degrees of difference. Moreover, when all stimulus dimensions are about equally obvious to the $\mathrm{Ss}$, a number of quantitative models may be tested with this type of task, including rather specific tests of the order of sampling hypotheses.

\section{Reference}

Lordahl, D. S. Hypothesis behavior in a multidimensional oddity problem. Psychon. Sci., 1965, 3, 229-230.

\section{Note}

1. This work was supported by grant no. MH 10277 from the National Institute of Mental Health, U. S. Public Health Service. 